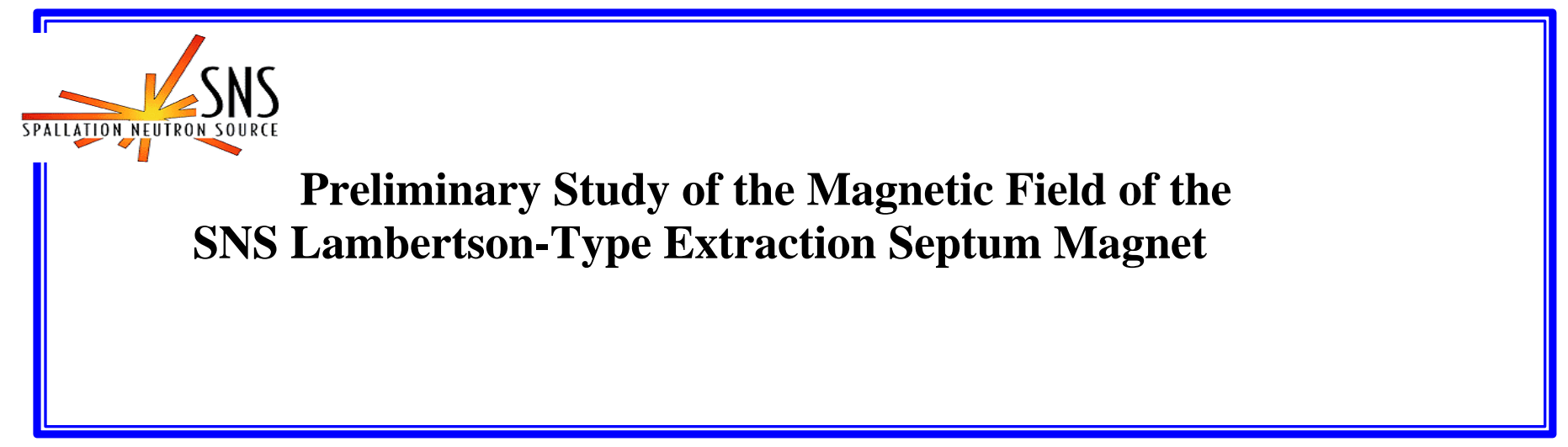

BNL/SNS TECHNICAL NOTE

NO. 054

N. Tsoupas, J. Brodowski, Y. Y. Lee, and J. Tuozzolo

December 22, 1998

ALTERNATING GRADIENT SYNCHROTRON DEPARTMENT BROOKHAVEN NATIONAL LABORATORY UPTON, NEW YORK 11973 


\title{
Preliminary Study of the Magnetic Field of the SNS Lambertson-type Extraction Septum Magnet
}

\author{
N. Tsoupas, J. Browdoski, Y.Y. Lee, J. Tuozzolo
}

\begin{abstract}
We present results from a preliminary study of the magnetic field that is generated by the SNS Lambertson-type extraction magnet.

The study is focused on the following items:

a) The optimization of the magnetic field uniformity of the magnetic field $\mathrm{B}_{\mathrm{y}}$ in the main field region well inside the magnet.

b) The optimization of the uniformity of the magnetic field integral $\int B_{y} . d l$ in the main field region along the various particle trajectories inside the magnet.

c) The minimization of the absolute value of the magnetic field in the circulating beam region.

The study was performed using the computer code opera3d (TOSCA) (Ref.1) which can perform three dimensional static magnetic field calculations.
\end{abstract}

\section{Geometrical characteristics of the magnet}

The beam extraction scheme (Ref. 2) of the SNS accumulator ring will employ a Lambertson-type extraction magnet which will bend the beam away from the ring and into the transfer line that leads to the SNS target. A cross section of the septum magnet is shown in Fig. 1. This figure shows, the upper and lower pole pieces between which the main magnetic field is generated to bend the extracted beam, the return yoke, the current carrying coil, and the beam pipe (almost elliptical in shape) of the circulating beam. A 3-dimensional view of the upstream section of the septum magnet which is closer to the SNS accumulator ring, is shown in Fig. 2a (side view) and Fig. 2b (side and under view).

This geometry of the septum magnet as shown in Figs $2 \mathrm{a}$, and $2 \mathrm{~b}$ does not lend itself to 2-dimensional magnetic field calculations because that can not provide reasonable results about the magnetic field at the entrance (also exit) of the septum magnet. It is therefore necessary that 3-dimensional magnetic field calculations are performed to determine the magnetic field at the entrance and exit of the septum magnet. The results of the 3dimensional magnetic field calculations which are presented in this technote are based on the magnet geometry shown in Figs 2a, and 2b. 


\section{Field requirements in the various regions of the magnet}

The goal of the final design study is to obtain a model septum magnet which satisfies the required field uniformity in the main field region and also minimizes the absolute value of the magnetic field in the circulating beam region.

The required value of the field uniformity in the main field region, inside the magnet at the region occupied by the beam is set to be $\Delta \mathrm{B} / \mathrm{B} \sim 10^{-3}$. This value keeps all the multipoles, including the quadrupole, at a low enough level to make the beam coupling insignificant.

In addition to the magnetic field uniformity well inside the magnet, the magnetic field integral uniformity $\left\{\Delta\left(\int \mathrm{B}_{\mathrm{y}} \cdot \mathrm{dl}\right) /\left(\int \mathrm{B}_{\mathrm{y} 0} \cdot \mathrm{dl}\right)\right\}^{1}$ which is calculated along the particle trajectories in the septum magnet (including the end effects) should be about $10^{-3}$. This may require special shaping of the pole faces at the entrance and exit of the septum magnet.

The absolute value of the magnetic field in the circulating beam pipe of the SNS accumulator ring has been set to be below 5 Gauss. This value of the magnetic field will not perturb significantly the circulating beam during the storage of the beam in the accumulator.

The results of this study will be the basis of additional magnetic field calculations to be performed on the actual septum magnet which may differ in size from the magnet size shown in Figs. 2a, and 2b.

\section{Set up of 3-D calculations}

The model of the septum magnet used in these calculations is shown in Figs. $2 \mathrm{a}$, and $2 \mathrm{~b}$ and represents only $40 \mathrm{~cm}$ of total length of the magnet at the entrance. This length is four times the gap of the magnet $(10 \mathrm{~cm})$ and is adequate to study the magnetic field behavior well inside the magnet and at the entrance/exit fringe field regions.

The computer code used to perform the electromagnetic field calculations is based on the finite element method and the computer code provides the flexibility to select regions of the magnet where more accurate calculations of the magnetic field is required. This can be done by adjusting the distance between the "nodes", in the various regions of the magnet. Thus in the main field region where the extracted beam is moving, the distance between the nodes is $2-3 \mathrm{~mm}$ in the transverse to the beam direction. The "node" distance in the longitudinal to the beam direction is about $5 \mathrm{~cm}$ well within the magnet, and about $5 \mathrm{~mm}$ at the fringing field region. The total number of finite elements is $\sim 650000$.

The geometry of the magnet does not depict any symmetry and the default boundary conditions (that the code provides) were assigned at the boundaries which were placed at $\sim 3 \mathrm{~m}$ away from the magnet.

\footnotetext{
${ }^{1}$ The quantity $\Delta\left(\int \mathrm{B}_{\mathrm{y}} \cdot \mathrm{dl}\right)=\left\{\int \mathrm{B}_{\mathrm{y}} \cdot \mathrm{dl}-\int \mathrm{B}_{\mathrm{y} 0} \cdot \mathrm{dl}\right\}$ where $\int \mathrm{B}_{\mathrm{y}} \cdot \mathrm{dl}=$ field integral along a particle trajectory and $\int \mathrm{B}_{\mathrm{y} 0} \cdot \mathrm{dl}=$ field integral along the central particle trajectory.

${ }^{2}$ In this computer code a "node" is defined as a point where the field can be calculated exactly. The magnetic field at any other point between the nodes is calculated either by linear or quadratic interpolation.
} 


\section{Presentation of results}

The results of this study are preliminary and its purpose is twofold; first to determine whether this particular magnet design (H-frame) can meet the magnetic field requirements mentioned above, and second to help us devise modifications to the magnet in order to meet these requirements.

More specifically, the study will answer the following questions:

a) What is the magnitude of the magnetic field in the circulating beam region?

b) Can we find ways to reduce the magnetic field in this region to acceptable levels?

c) For the specific magnet geometry shown in Figs. 2a, and 2b what is the field uniformity in the main field region, well within the magnet at the region occupied by the extracted beam?

d) Can we improve the field uniformity by shimming the edges of the upper pole piece as shown in Fig. 2b?

e) What is the uniformity of the integral magnetic field along the particle trajectory?

f) Can we improve the integral magnetic field uniformity by modifying the entrance and exit pole faces?

g) Is there an alternative magnet geometry (eg. window frame design) which provides an improved magnetic field, and integral magnetic field uniformity ?

\section{The Magnetic field in the region of the circulating beam}

The modulus of the magnetic field $\mathrm{B}_{\text {mod }}$ in the circulating beam region (elliptical area shown in Fig. 1) is plotted in Figs. $3 a$ and $3 b$.

Figure 3 a shows the $\mathrm{B}_{\text {mod }}$ inside the circulating beam region, and over a rectangular area with the following coordinates:

a) $y=-3 \mathrm{~cm}$. The rectangle is parallel to the bottom pole face of the magnet.

b) $(\mathrm{x}=+-3 \mathrm{~cm}, \mathrm{z}=0 \mathrm{~cm})$ The lateral extend of one side of the rectangle is $\mathrm{x}=+-3 \mathrm{~cm}$ and it runs from well within the magnet $(\mathrm{z}=0 \mathrm{~cm})$.

c) $(x=+-3 \mathrm{~cm}, \mathrm{z}=60 \mathrm{~cm})$ The corresponding parallel side ends at $\mathrm{z}=60 \mathrm{~cm}$.

The magnitude of the magnetic field over this rectangle is lower than 5 Gauss. This low magnetic field value is due to the shielding effect of the return yoke of the magnet as well as to the extension of the lower pole face by a distance of $20 \mathrm{~cm}$ beyond the end of the upper pole piece (see Figs 1a,1b).

Figure $3 \mathrm{~b}$ is a similar plot of the $\mathrm{B}_{\text {mod }}$ over the same rectangle as the plot of figure $3 \mathrm{a}$, but for a magnet without the additional extension of the bottom pole face (see Fig. 5).

The strong shielding effect which is provided by the extension of the lower pole face can be demonstrated by comparing Fig. 3a with Fig. $3 b$.

The units of the magnetic field shown in Figs 3a, and 3b, is in Gauss and the distance in $\mathrm{cm}$.

\section{The Magnetic field in the region of the extracted beam}

The magnetic field in the region of the extracted beam should contain low values of high order multipoles in order to keep at a minimum any beam coupling between the 
horizontal and vertical beam parameters ${ }^{3}$. Separate studies on the effect of the magnetic field uniformity on the beam coupling, showed that a beam uniformity $\Delta \mathrm{B}_{\mathrm{y}} /\left(\mathrm{B}_{\mathrm{y}}\right)_{0} \sim 10^{-3}$ introduces multipoles which have practically no contribution to the beam coupling. We can reduce the magnitude of the various multipoles for the region inside the magnet by increasing the pole width and properly shimming the edges of the upper pole piece. It is clear that one cannot avoid the contribution of the various multipoles at the entrance and exit of the magnet. For this reason we will consider the field homogeneity of the magnet at the entrance and exit, separately from the field homogeneity well inside the magnet.

\section{Magnetic field well inside the magnet}

For an $\mathrm{H}$-frame type magnet the field uniformity well within the magnet is practically independent of the ratio $\mathrm{g} / \mathrm{w}$ ( $\mathrm{g}=$ gap of the magnet, $\mathrm{w}=$ width of the magnet pole) when $\mathrm{g} / \mathrm{w}<<1$. For our particular model however (see Fig. 1) the field uniformity depends strongly on the $\mathrm{g} / \mathrm{w}$ ratio because $\mathrm{g} / \mathrm{w}=0.5$.

Fig. 4a shows a plot of the component $\mathrm{B}_{\mathrm{y}}$ over a rectangular area which is located inside the main field region of the magnet shown in Figs. 2a. This rectangular area is parallel to the pole faces at distance $y=2 \mathrm{~cm}$ above the bottom pole face. The lateral extend of the rectangle is $+-3 \mathrm{~cm}$ and the side of the rectangle along the beam direction is $5 \mathrm{~cm}$. The coordinates of the rectangle appear in the right hand side of the plot, and are all in $\mathrm{cm}$.

Fig. $4 \mathrm{~b}$ shows a similar plot of the $\mathrm{B}_{\mathrm{y}}$ component over a rectangle which is a distance $y=3 \mathrm{~cm}$ from the bottom pole face but otherwise having the same coordinates as the rectangle in Fig. 4a.

Comparison of the $\mathrm{B}_{\mathrm{y}}$ components which appear in the figures $4 \mathrm{a}$ and $4 \mathrm{~b}$ provides us with a measure of the field uniformity in the vertical direction.

Figures $6 \mathrm{a}$ and $6 \mathrm{~b}$ plot the same quantities as figures $4 \mathrm{a}$ and $4 \mathrm{~b}$, but for a magnet without the shims at the edges of the upper pole pieces (see magnet in Fig. 5 which has no shims at the edges of the pole pieces).

\section{Magnetic field integral uniformity along the particle trajectories.}

This section discusses the magnetic field at the entrance of the magnet.

Figure 7 shows the $\mathrm{B}_{\mathrm{y}}$ component of the magnetic field over a rectangle parallel to the pole pieces at a distance $2 \mathrm{~cm}$ from the bottom pole piece. This rectangle starts well inside the magnet and extends well outside the magnet (see $\mathrm{x}, \mathrm{y}, \mathrm{z}$ coordinates of the rectangle at the right hand side of figure 7 ), and provides a good view of the $\mathrm{B}_{\mathrm{y}}$ field component at the entrance of the magnet.

It is clear that the magnetic field at the entrance/exit of the magnet is not uniform, especially along the beam direction, and the beam coupling due to the various multipoles is unavoidable. It is necessary however to ensure that all the particles passing through the

\footnotetext{
${ }^{3}$ The lack of the median plane symmetry of this magnet may also cause linear coupling due to any significant quadrupole multipole (skew quadrupole).
} 
magnet are subject to the same bending angle. An exact measure of the angle of bend is the magnetic field integral over the various particle trajectories. The calculated uniformity of the magnetic field integral along the various particle trajectories is better than $10^{-3}$ for all particles that pass through a square with sides of $6 \mathrm{~cm}$. The center of the square is located a point with coordinates $(x, y)=(0,3)$.

\section{Conclusions}

The desired magnetic field uniformity $\Delta \mathrm{B}_{\mathrm{y}} / \mathrm{B}_{\mathrm{y} 0} \sim 10^{-3}$ (well inside the magnet) over a distance of +- $6 \mathrm{~cm}$ transverse to the beam direction, can be achieved by increasing the width of pole pieces and adjusting the shims at the edges of the upper pole pieces. Having achieved the required magnetic field uniformity $\Delta \mathrm{B}_{\mathrm{y}} / \mathrm{B}_{\mathrm{y} 0} \sim 10^{-3}$ inside the magnet, the required magnetic field integral uniformity $\left\{\Delta\left(\int \mathrm{B}_{\mathrm{y}} \mathrm{dl}\right) /\left(\int \mathrm{B}_{\mathrm{y} 0} . \mathrm{dl}\right)\right\} \sim 10^{-3}$ will be easily satisfied for a magnet with length many times its gap as is this case.

\section{References}

1. VECTOR FIELDS Inc.

2. Spallation Neutron Source Design Manual June 1998 E. Blesser BNL

\section{Figure Captions}

Figure 1. Cross section of the septum magnet normal to the beam direction. This cross section is taken at the entrance of the magnet. In all the figures the distances are in $\mathrm{cm}$.

Figure 2a. 3-Dimensional view of the entrance of the magnet (top and side view). Note the extension of the bottom pole piece of the magnet, in reference to the top pole piece.

Figure 2b. 3-Dimensional view of the entrance of the magnet (under and side view).

Figure 3a. Plot of the $\mathrm{B}_{\text {mod }}$ component of the magnetic field inside the pipe of the circulating beam region. The values of the $\mathrm{B}_{\text {mod }}$ are calculated over a rectangle with $(\mathrm{x}, \mathrm{y}, \mathrm{z})$ coordinates shown in the right hand side of the plot. In all the plots the units of the magnetic field are in Gauss. The plotted $\mathrm{B}_{\text {mod }}$ component corresponds to the magnet which has the bottom pole piece extended by $20 \mathrm{~cm}$ (Fig. 2a).

Figure $3 \mathrm{~b}$. Same as Figure $3 \mathrm{a}$ but the plotted $\mathrm{B}_{\text {mod }}$ component corresponds to the magnet which has no iron extension at the bottom pole piece. (see Fig. 5). 
Figure 4a. Plot of the $\mathrm{B}_{\mathrm{y}}$ component of the magnetic field inside the magnet over a rectangle which is parallel to the bottom pole face of the magnet and at a distance of $2 \mathrm{~cm}$. The $(\mathrm{x}, \mathrm{y}, \mathrm{z})$ coordinates of the rectangle appear in the right hand side of the plot.

Figure $4 \mathrm{~b}$. Same as in Figure $4 \mathrm{a}$ but over a rectangle at a distance of $2 \mathrm{~cm}$ from the bottom pole face.

Figure 5. 3-Dimensional view of the entrance of the magnet (bottom and side view). Note that, unlike Fig. 2a, there is no extension of the bottom pole piece of the magnet There is also no shimming at the edges of the upper pole piece.

Figure 6a. Same as in Figure 4a but for the magnet shown in Fig. 5 (no pole pice extension)

Figure 6b. Same as in Figure 4b but for the magnet shown in Fig. 5 (no pole pice extension)

Figure 7. Plot of the $\mathrm{B}_{\mathrm{y}}$ component of the magnetic field over a rectangle which is parallel to the bottom pole piece and extends well outside the magnet. The (x,y,z) coordinates of the rectangle appear in the right hand side of the plot. 


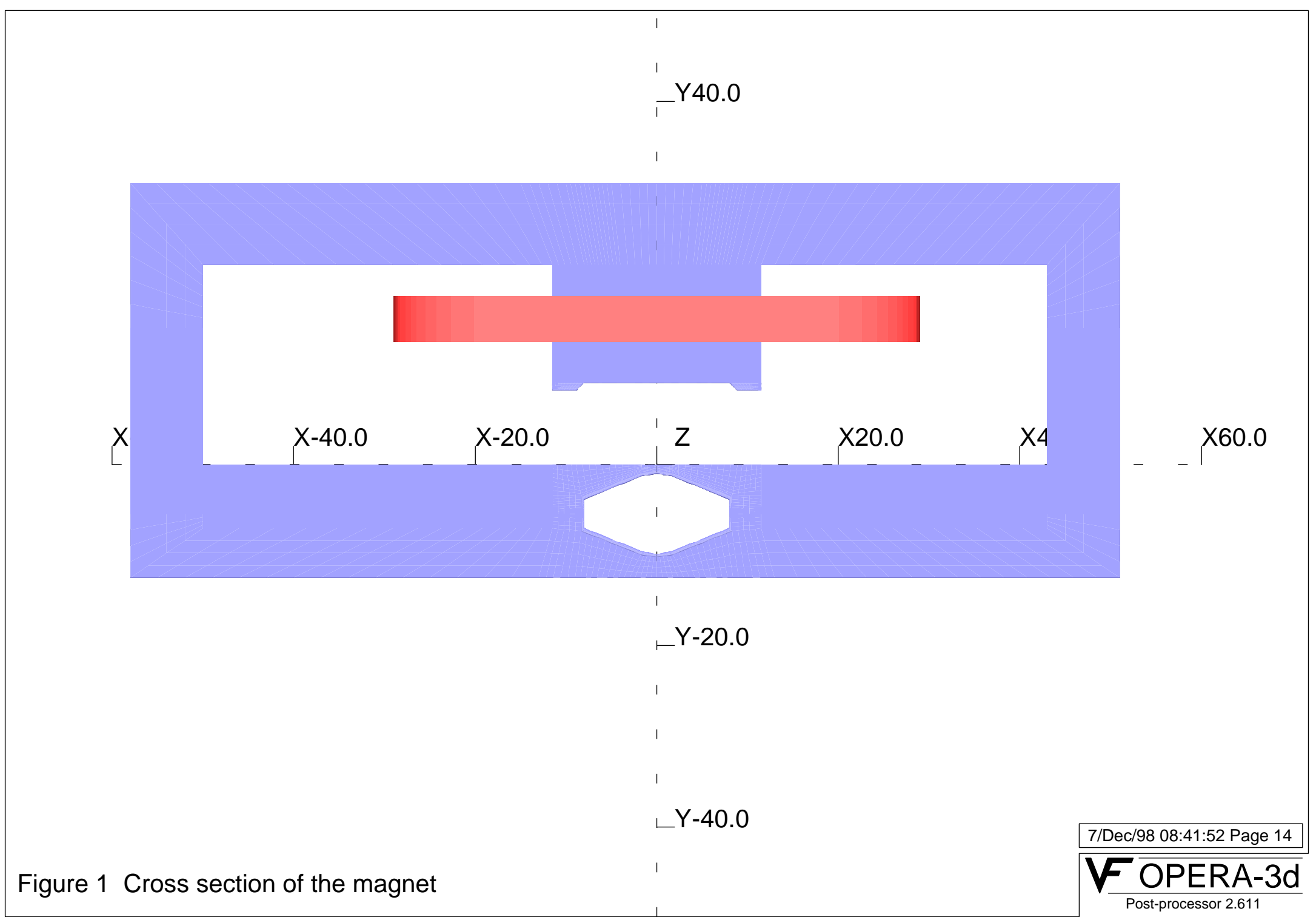




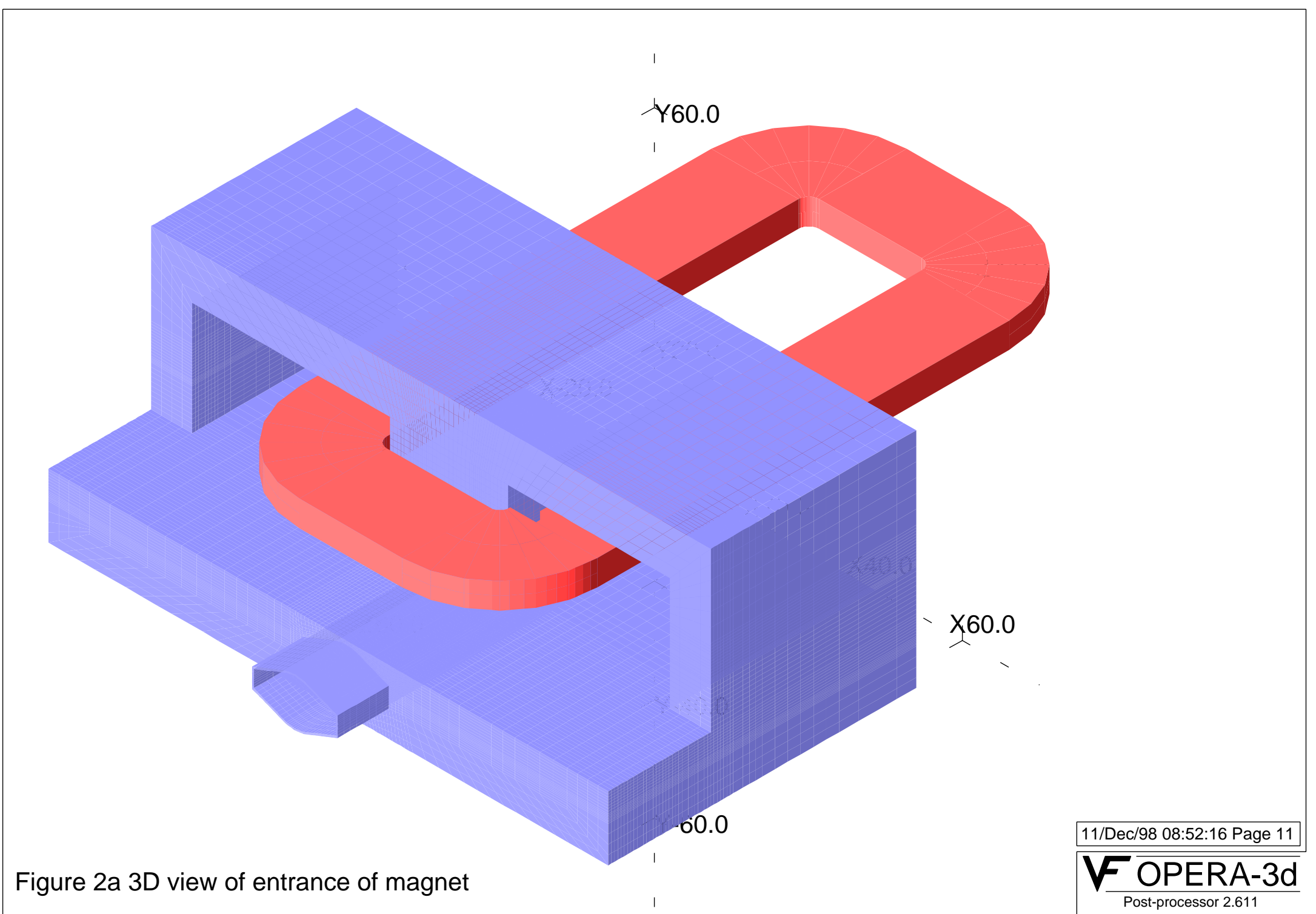




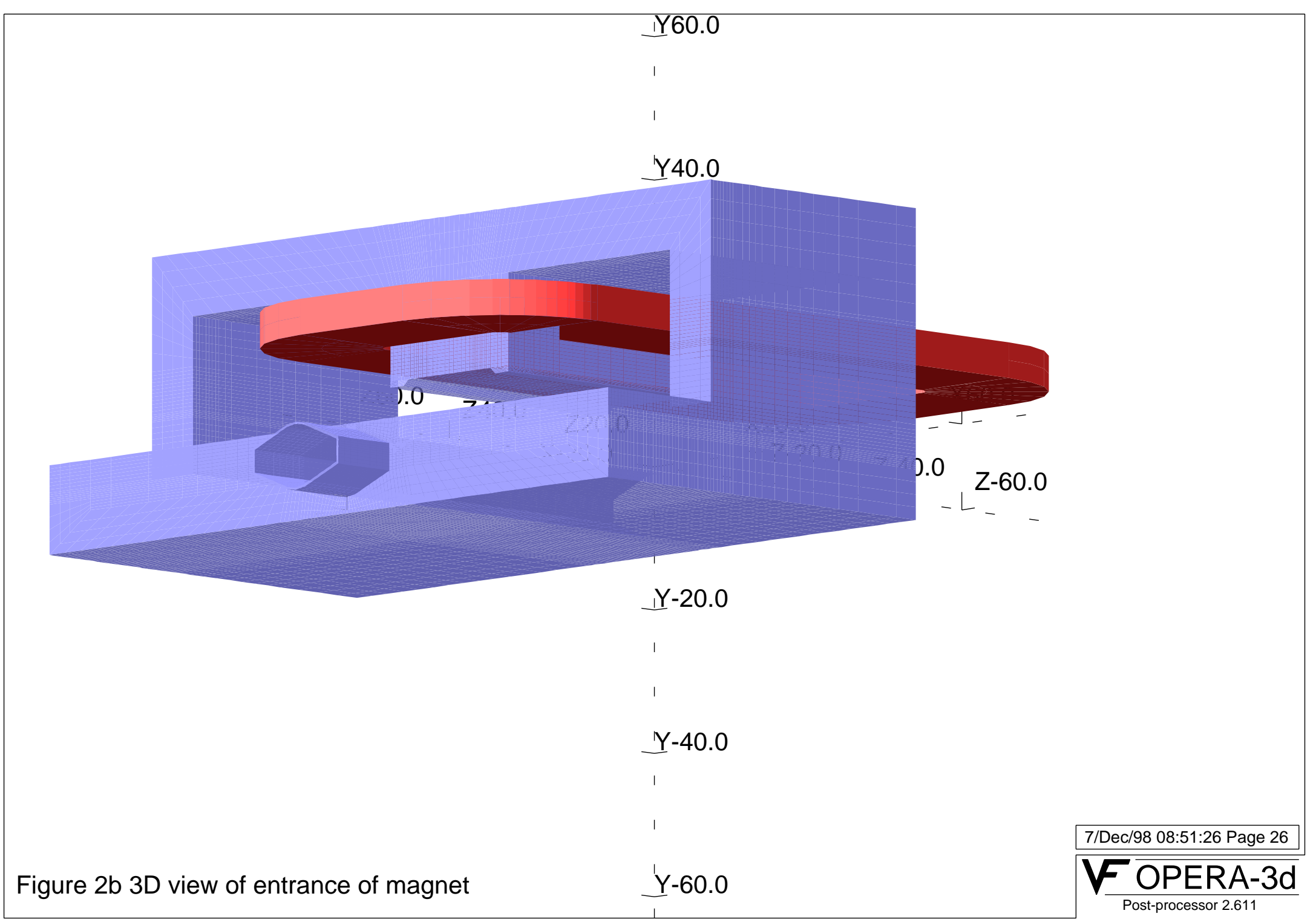




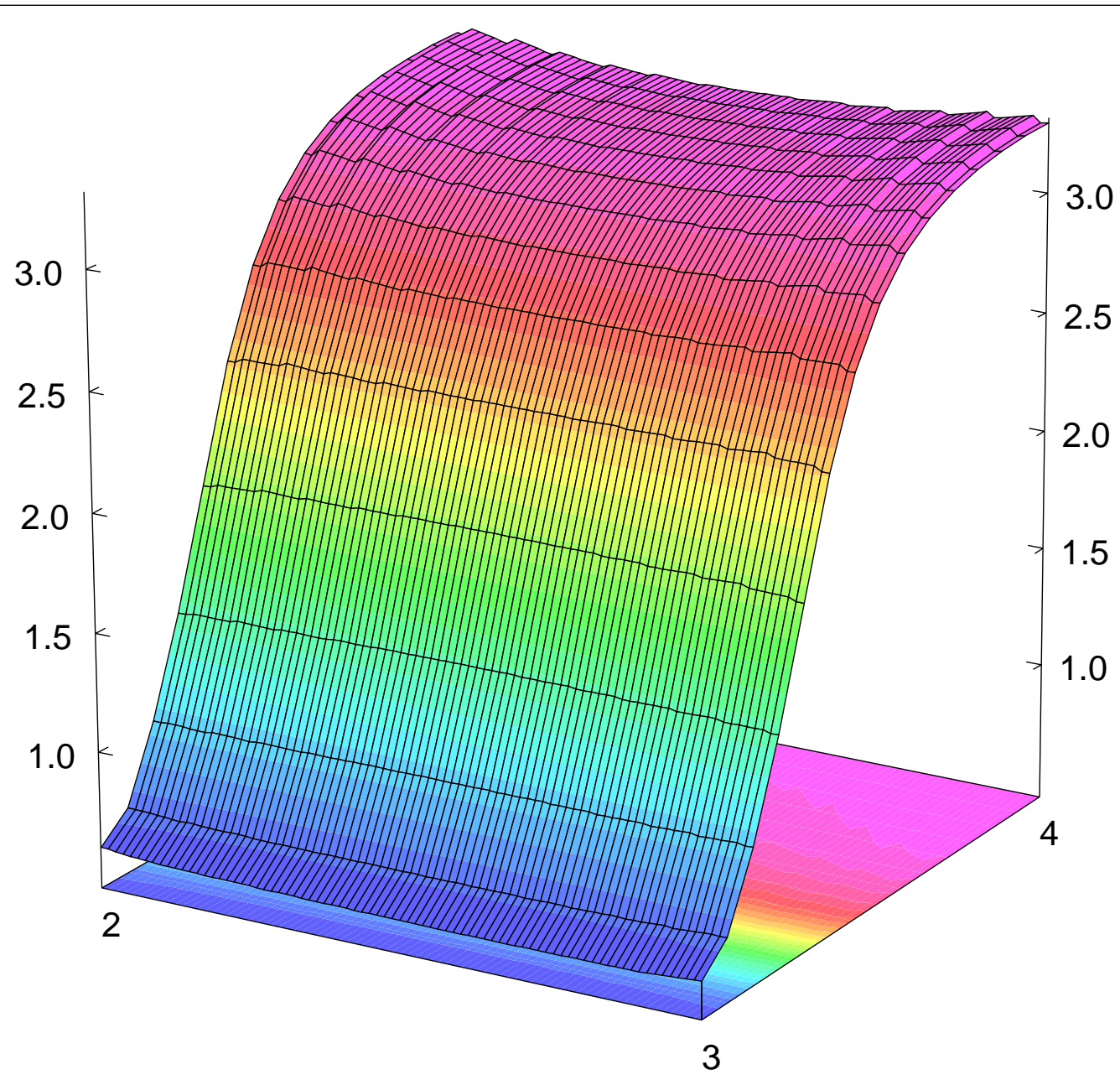

$$
\begin{gathered}
1=-3.0 \\
-3.0 \\
0.0 \\
2=-3.0 \\
-3.0 \\
60.0 \\
3=3.0 \\
-3.0 \\
60.0 \\
4=3.0 \\
-3.0 \\
0.0 \\
\text { Cartesian }
\end{gathered}
$$

0

Minimum $=0.562259$, Maximum $=3.31333$

Integral = 873.632

Figure 3 a Bmod In Pipe of Circul. beam 


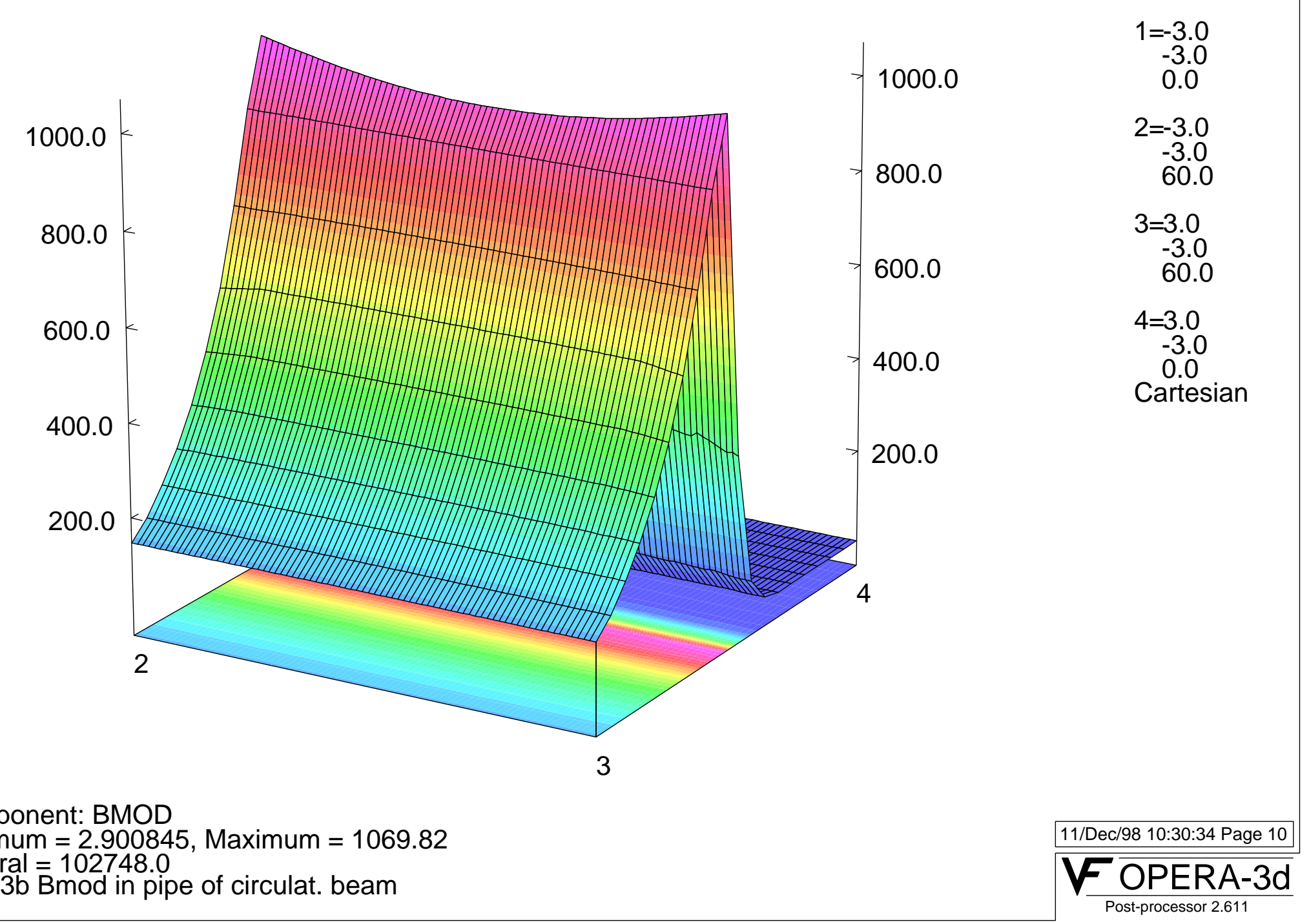




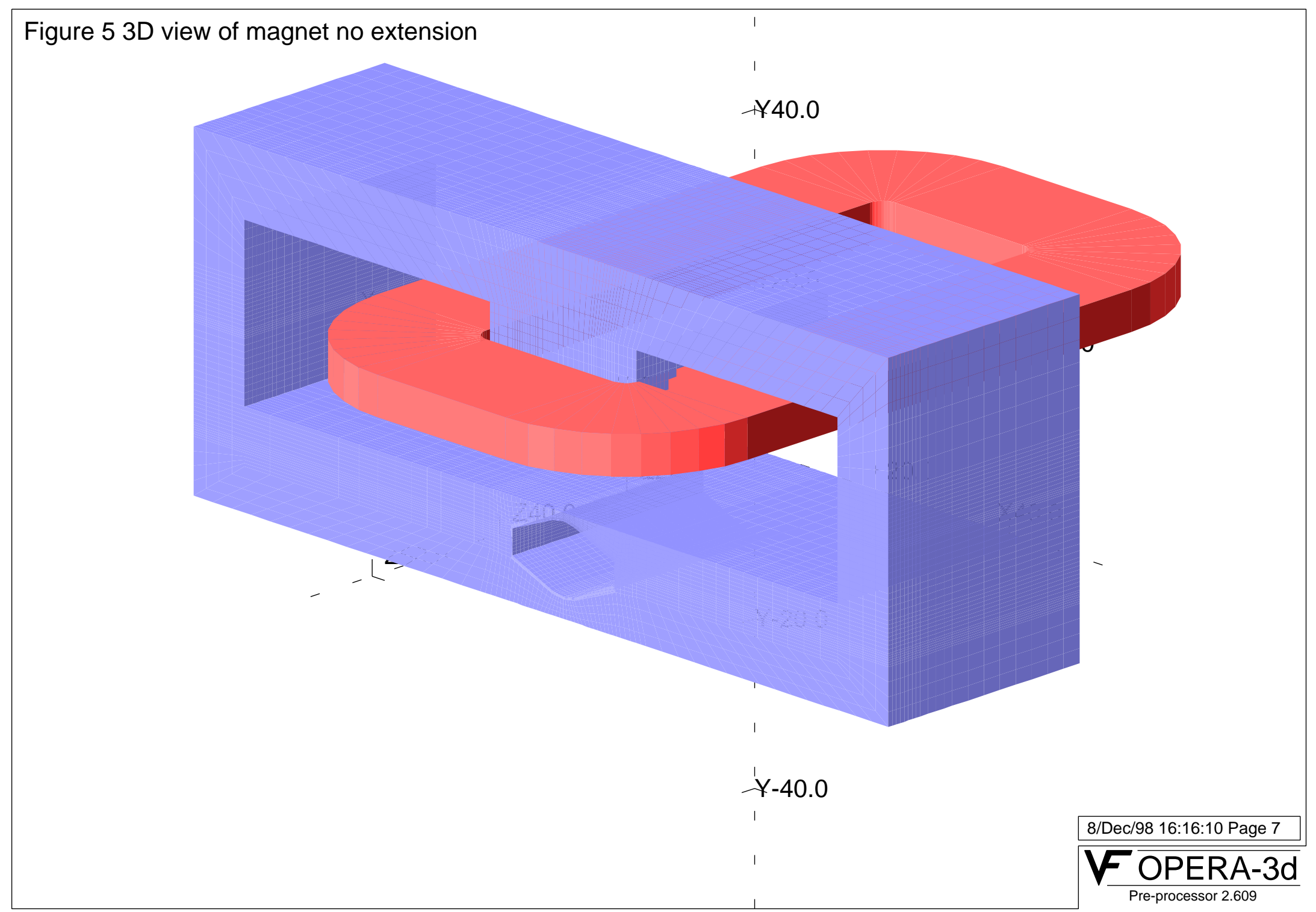




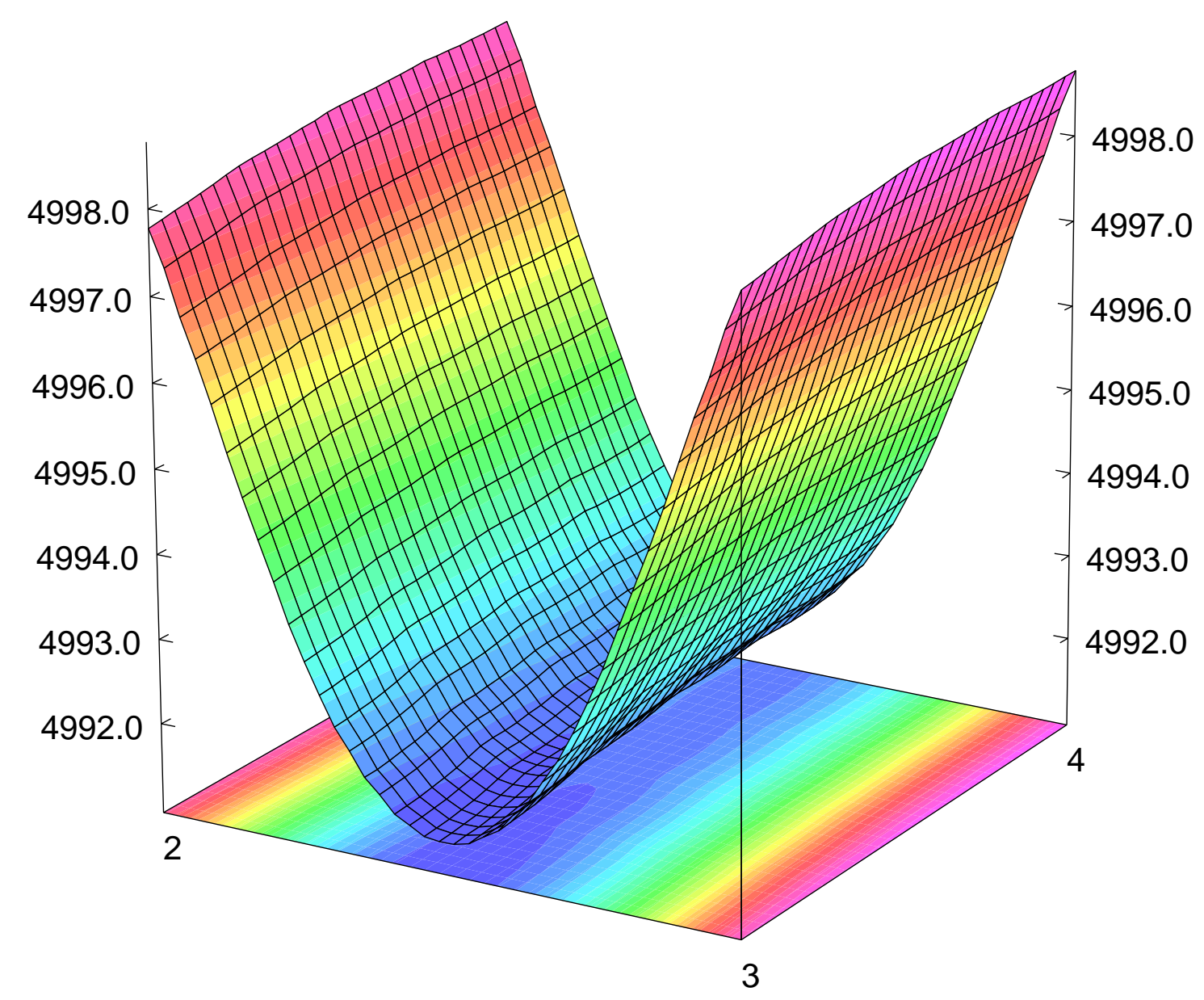

$1=-4.0$

2.0
0.0

$=-4.0$ 2.0

$3=4.0$ 2.0

$$
15.0
$$

$4=4.0$

2.0
0.0

Cartesian

Component: BY

Minimum $=4991.31$, Maximum $=4998.76$

Integral = 599291.0

'Figure 4a By component inside magnet 


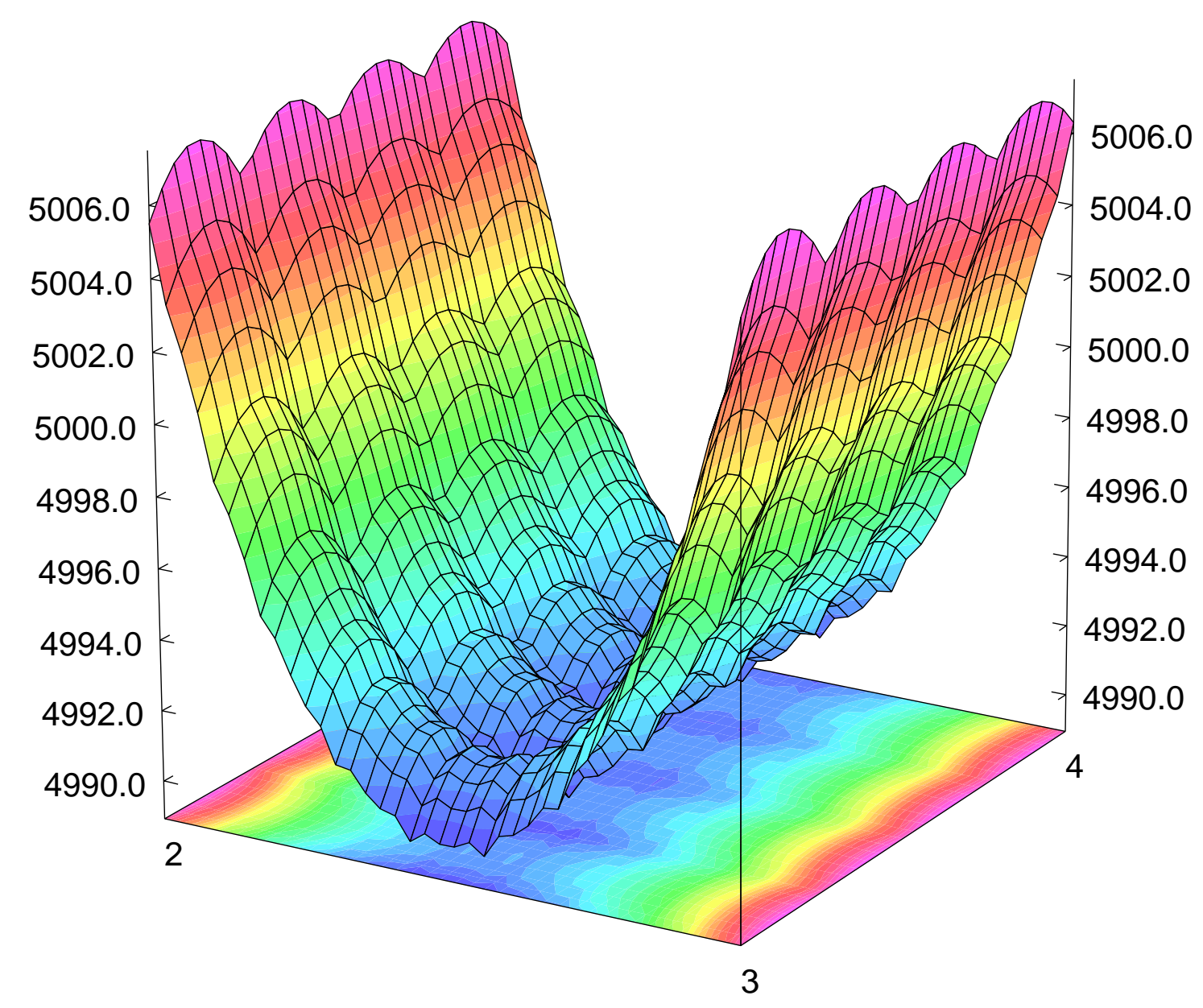

$1=-4.0$

3.0
0.0

$2=-4.0$

3.0

15.0

$3=4.0$

3.0

15.0

$4=4.0$

3.0

Cartesian

Component: BY

Minimum $=4989.81$, Maximum $=5007.5$

Integral = 599503.0

Figure $4 \mathrm{~b}$ By component inside magnet

11/Dec/98 11:35:17 Page 27

F OPERA-3d

Post-processor 2.611 


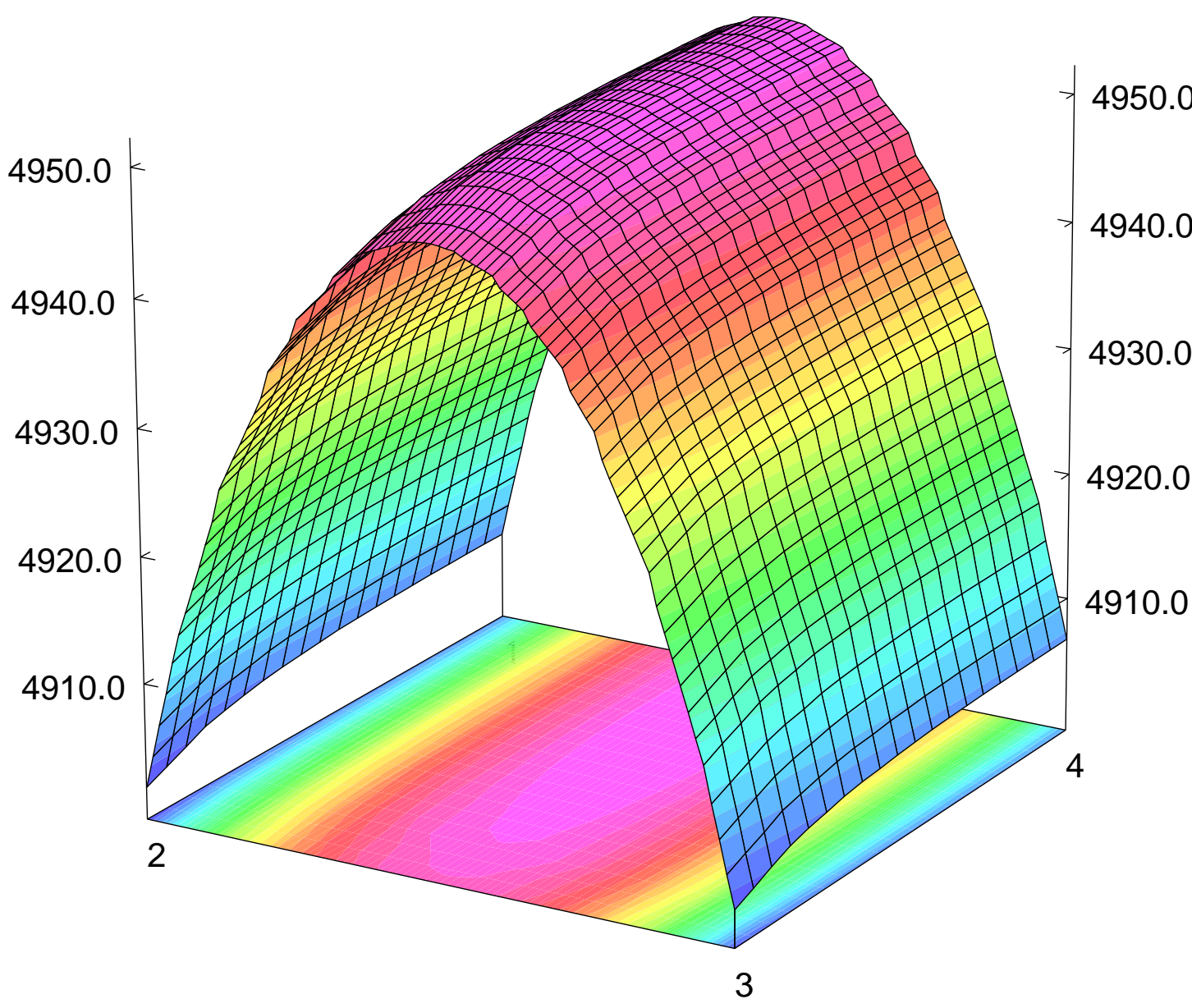

$1=-4.0$

2.0

$2=-4.0$ 2.0
15.0

$3=4.0$

2.0

$4=4.0$

2.0

Cartesian

Component: BY

Minimum $=4901.79$, Maximum $=4952.26$

Integral $=592436.0$

Figure6a By component inside the magnem

11/Dec/98 11:42:26 Page 15

F OPERA-3d

Post-processor 2.611 


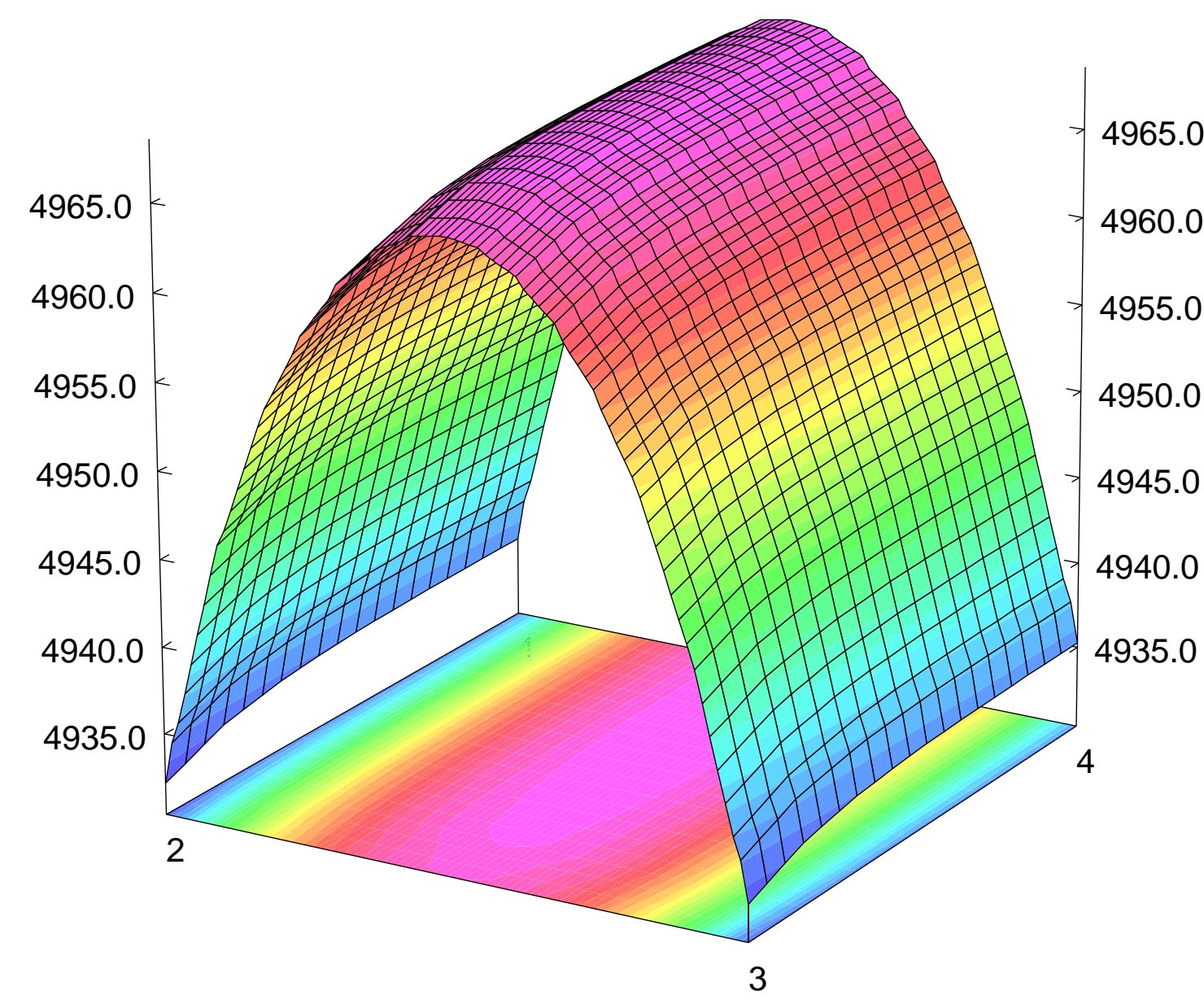

$1=-4.0$

3.0

0.0

$2=-4.0$

3.0
15.0

$=4.0$

3.0

15.0

$4=4.0$

3.0

Cartesian

Minimum $=4932.16$, Maximum $=4968.53$

Integral $=594919.0$

Figure6b By component inside the magnem

11/Dec/98 11:40:43 Page 13

V OPERA-3d

Post-processor 2.611 


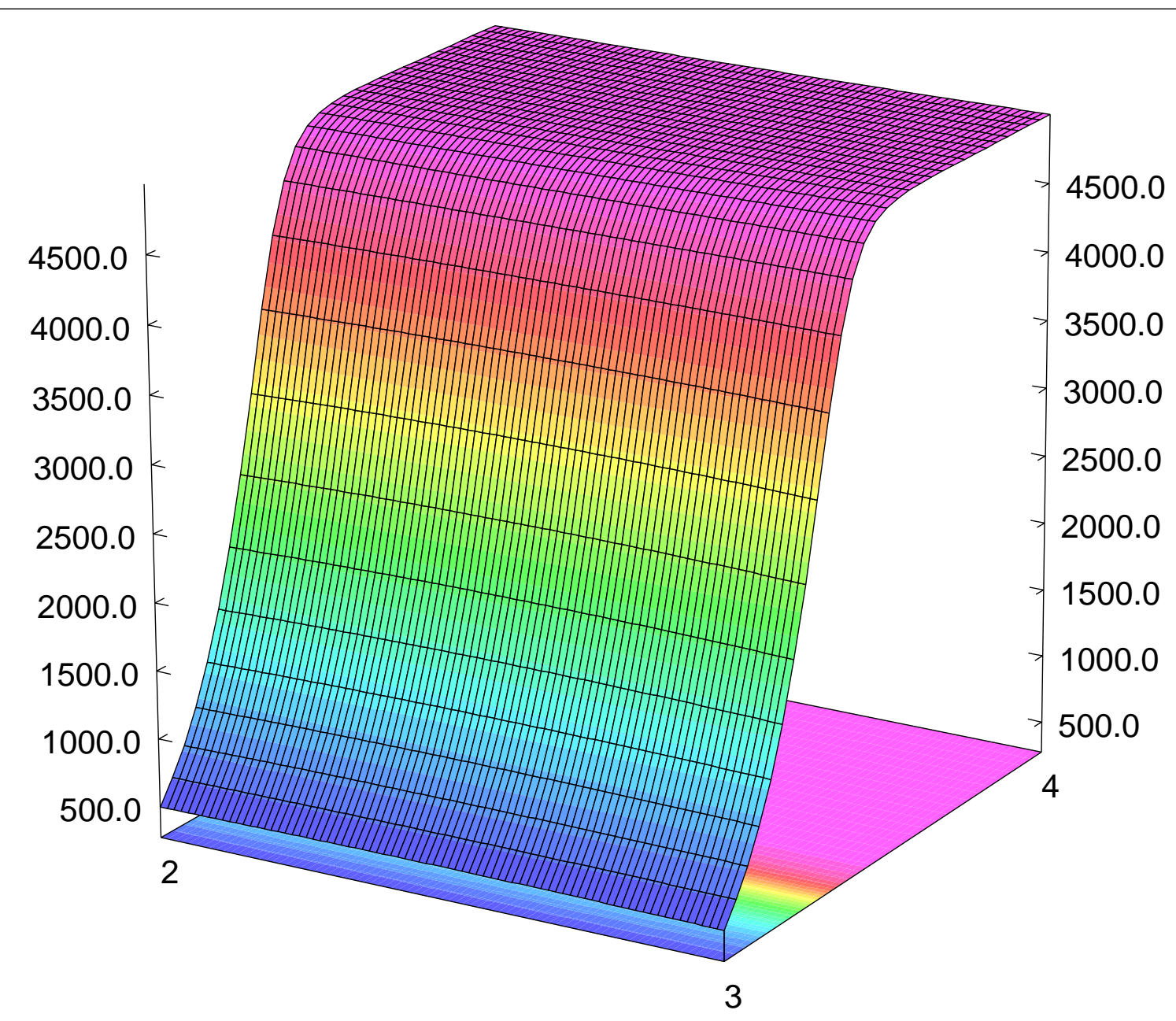

$1=-4.0$

2.0
0.0

$2=-4.0$ 60.0

$3=4.0$ 2.0 60.0

$4=4.0$

2.0

Cartesian

Component: BY

Minimum $=496.171$, Maximum $=4998.76$

Integral $=1877724.0$
Figure 7 By comp. including edge eff. 